\title{
GIANT RADIO PULSES FROM PULSARS
}

\author{
ASHOK K. SINGAL \\ Astronomy and Astrophysics Division, Physical Research Laboratory, Navrangpura, \\ Ahmedabad-380009, India; E-mail: asingal@prl.ernet.in
}

\begin{abstract}
We present some results from our observations of giant pulses from PSR B0950+08 at $103 \mathrm{MHz}$. These observations, now extending over a year, have shown the highest rate of occurrence of giant pulses seen from any known pulsar. Large fluctuations in the intensity levels of individual giant pulses and in their occurrence rate per unit interval of time are seen during a single day's observations, as well as from one day to the next. We conclude that these intensity variations are likely to be intrinsic to the pulsar.
\end{abstract}

\section{Introduction}

Today about 1000 pulsars are known, having their pulse periods between a few milliseconds to a few seconds. At radio wavelengths, most pulsars show enormous variations between successive individual pulses, however, the variations usually disappear when a sufficient number of pulses are superimposed and added together to make an integrated profile. The shapes of the integrated profiles are quite stable and easily reproducible, but they often show amplitude variations on various time scales. These variations have been ascribed to ISM diffraction and refraction scintillation effects depending upon the time scale of variations. The pulse to pulse intensity variations typically lie within an order of magnitude of the average pulse strength, except for the nulling seen in some cases where the pulse intensity may drop below one percent level of the mean pulse strength. However, in a few cases, the pulse intensity sometimes exceeds the mean pulse strength much more than an order of magnitude (Lundgren et al., 1995; Cognard et al., 1996). Such are known as giant pulses. Statistical knowledge about the occurrence frequency of such giant pulses is still lacking, partly because it requires a fairly large amount of precious telescope time spread over many months or even longer, to monitor such sources on a regular basis to get their temporal statistics. We have recently begun a longterm monitoring programme for PSR B0950+08 and a couple of other pulsars at $103 \mathrm{MHz}$, by observing them daily for half an hour. The aim is to gather statistical information on the occurrence frequency of individual giant pulses as well as to study the long term variations in the daily-average pulse intensities. 


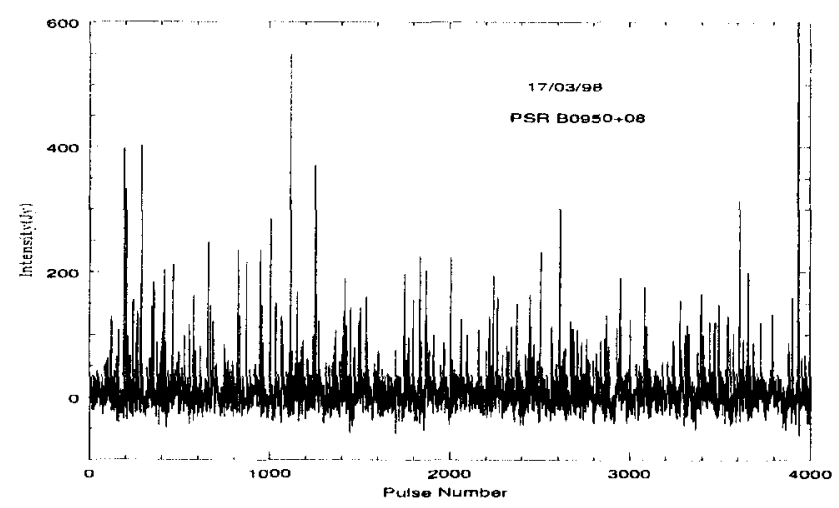

Figure 1. A sample of giant pulses observed from PSR B0950+08 at $103 \mathrm{MHz}$.

\section{Observations}

Our observations were made with the Rajkot radio telescope, situated at a location (longitude $70.7^{\circ} \mathrm{E}$, latitude $22.3^{\circ} \mathrm{N}$ ) in the western parts of India. The telescope is a transit instrument, consisting of 1024 dipoles spread over a 5000 square metre area. The telescope, designed in 1970s basically for the interplanetary scintillation (IPS) studies, operates at a centre frequency of $103 \mathrm{MHz}$, with a bandwidth of $1.6 \mathrm{MHz}$. The wide east-west beam $\left(8^{\circ}\right)$ of the telescope allows an object near the meridian to be studied for about 32 minutes, the north-south pointing is done using the phased array technique. Over a year of observations, data for more than a million pulses have been recorded for PSR B0950+08. While most individual pulses will be too weak to be detected (signal to noise $\lesssim 0.2$ ), single giant pulses may greatly exceed the noise level. We have chosen 20 times the mean pulse intensity as the threshold for a pulse to be called giant.

\section{Results and Discussion}

The giant pulses have earlier been reported for only two, relatively fast, pulsars, namely Crab pulsar (Lundgren et al., 1995) and PSR B1937+21 (Cognard et al., 1996). The number of giant pulses seen from these pulsars vary. While one out of 383 pulses from Crab pulsar was found to be giant (Lundgren et al., 1995), the millisecond pulsar B 1937+21 showed one pulse in 10000 to be more than 20 times the mean 'pulse on' flux density (Cognard et al., 1996). From our data for about one million pulses for B0950+08, roughly one percent were found to be giant pulses, and at least one in 10000 crossed the mean pulse level by a factor of 100 , with some individual pulses as large as 300 times the mean. One very interesting feature of our data is that the giant pulses are not distributed uniformly even in a rough manner. Though sometimes a few giant pulses may follow each 
other in quick succession, but more than two thirds of the time they appear alone where immediately next to a giant pulse the neighbouring pulses may be more than order of magnitude weaker or could even fall below our detection limit (implying a fluctuation in flux-density $\gtrsim 50$ ). Then there is a wide fluctuation in the giant pulse occurrence frequency from one day to the next. In fact almost all ( $\gtrsim 99 \%$ ) of the total giant pulses observed by us have been seen during 'active' days, which are about one fourth of the total days of observations. There are days when as much as $10 \%$ of the total pulses are giants, while many other days show only 'nulls' when no detectable emission is seen either in the power spectrum or in the folded pulse data. Hardly any unambiguous giant pulse is seen on these extremely quiet days and the day's mean pulse intensity has an upper limit of $\sim 0.3 \mathrm{Jy}$, our sensitivity limit for 32 minutes of observations.

The variations of the integrated pulse intensities on various time scale have generally been explained by invoking diffractive and refractive scintillation effects of the interstellar medium (ISM). In particular, the long term variations (on time scales of days and longer) have been explained in terms of the refractive effects (Rickett, Coles and Bourgois, 1984). The time scale of such variations for PSR B0950+08 at $103 \mathrm{MHz}$ is expected to be around 47 days, translating from the calculations made earlier for $74 \mathrm{MHz}$ (Gupta, Rickett and Coles, 1993). This is more than an order of magnitude higher value than the value actually observed where many times the daily average from one day to the next rises from about $\lesssim 0.3$ to $\sim 20$ Jy (a factor of $\gtrsim 50$ ) within a single day's time. The extremely large pulse to pulse amplitude fluctuations (i.e., the individual giant pulses) are undoubtly an intrinsic effect as the time scales for these are too short for these to be either diffractive or refractive scintillation effects, more so as our recent observations with Westerbork Radio Telescope, using the new pulsar machine (in collaboration with Richard Strom) have often shown individual extremely bright pulses that remain unresolved even with millisecond time resolutions. The day to day intensity variations also happen to be one of the most extreme in this pulsar. Now if the latter effect were to be ascribed to the interstellar refraction scintillations, it will imply that both the extreme cases of variations, intrinsic (giant pulses from the pulsar) and extrinsic (interstellar refraction scintillation effects), arising from two totally unconnected, independent phenomena, happen to occur in one and the same object (PSR B0950+08) out of so many known pulsars, something highly improbable. Though one cannot completely rule out the scintillations as their cause, it is much more likely that the daily variations too are intrinsically connected with the pulsar radiation mechanism.

We do not yet understand how and why a pulsar emits giant pulses and also why this phenomenon is seen only in a few cases. Most likely it is the fluctuations in the number of charges taking place in the radiation process that gives rise to the fluctuations in the net radio emission of the pulse intensity. The lack of observable radio radiation during nulling is perhaps caused by the absence of a dominant coherence mechanism. It may be possible to argue that giant pulse emission is opposite of 
nulling of a pulsar in which case, say, bunching of radiating charges enhances, giving rise to a higher degree of coherence. Cognard et al. (1996) examined the period $P$ and the period derivative $\dot{P}$ for a few dozen strongest pulsars and estimated their magnetic field strengths at the light-cylinder, $B_{l c} \sim 3 \times 10^{8} P^{-2.5} \dot{P}^{0.5} \mathrm{G}$. They noted a rather high $B_{l c} \sim 10^{6} \mathrm{G}$ for both Crab $\left(P=33 \mathrm{~ms}, \dot{P}=10^{-12.4}\right.$ sec $\left.\sec ^{-1}\right)$ and $\mathrm{B} 1937+21\left(P=1.56 \mathrm{~ms}, \dot{P}=10^{-19} \mathrm{sec} \mathrm{sec}-1\right)$, an order of magnitude higher value than for their other pulsars. From this they suggested that the giant pulsation phenomenon may have something to do with the magnetic field strength at the light-cylinder radius. From the known parameters of B0950+08, $\left(P=253 \mathrm{~ms}, \dot{P}=10^{-15.6} \mathrm{sec} \mathrm{sec}^{-1}\right)$, we find $B_{l c}$ to be only $\sim 150$ Gauss, not at all a high value. Thus one can almost rule out the hypothesis that the giant pulse emission physics is in particular dependent on the magnetic field strength at the light-cylinder radius. What else could it be that yet remains to be seen.

\section{Acknowledgements}

I gratefully acknowledge the partial financial support received from the organizers of the IAU Colloquium 182 for attending it.

\section{References}

Cognard, I., Shrauner, J.A., Taylor, J.H. and Thorsett, S.E.: 1996, Giant radio pulses from a millisecond pulsar, Astrophys. J. 457, L81-L84.

Gupta, Y., Rickett, B.J. and Coles, W.A.: 1993, Refractive interstellar scintillation of pulsar intensities at $74 \mathrm{MHz}$, Astrophys. J. 403, 183-201.

Lundgren, S.C., Cordes, J.M., Ulmer, M., Matz, S.M., Lomatch, S., Foster, R.S. and Hankins, T.: 1995, Giant pulses from the Crab pulsar: a joint radio and gamma-ray study, Astrophys. J. 453, 433-445.

Rickett, B.J., Coles, W.A. and Bourgois, G.: 1984, Slow scintillation in the interstellar medium, Astron. Astrophys. 134, 390-395. 